\title{
Cinobufagin Enhances the Sensitivity of Cisplatin- Resistant Lung Cancer Cells to Chemotherapy by Inhibiting the PI3K/AKT and MAPK/ERK Pathways
}

Guangxin Zhang ( $\nabla$ m18768117196@126.com )

Second Hospital of Jinlin University

Xueshibojie Liu

Second Hospital of Jinlin university

Yicun Wang

Second Hospital of Jinlin unversity

Chengyan Jin

Second hospital of Jinlin unversity

Zhengyang Lu

Xi'an Jiaotong University Medical College First Affiliated Hospital

Hao Yang

Second hospital of Jinlin unversity

Ti Tong

Second Hospital of Jinlin unversity

\section{Research Article}

Keywords: lung cancer, cisplatin, drug resistance, traditional Chinese medicine, Cinobufagin

Posted Date: December 8th, 2021

DOI: https://doi.org/10.21203/rs.3.rs-1127820/v1

License: (c) (1) This work is licensed under a Creative Commons Attribution 4.0 International License. Read Full License 


\section{Abstract}

Background: Lung cancer patients always develop serious chemotherapy resistance after long-term use of cisplatin (DDP) treatment. It has been demonstrated that combination of DDP with other chemotherapy drugs may significantly reduce drug resistance. Cinobufagin (CB) showed potent antitumor effect against lung cancer. However, the relevance of CB and DDP resistance in lung cancer remains unclear. This article will study the effects of $\mathrm{CB}$ on reversing lung cell resistance in vitro and in vivo.

Materials and Methods: The cell viability was evaluated using the Cell Counting Kit 8 (CCK8) assay. The apoptosis was detected by flow cytometry analysis and TUNEL staining. The invasiveness was detected by Invasion assay. The mRNA and protein of apoptosis-related proteins, P-AKT, P-PI3K, P-MEK1/2, PERK1/2 and MRP1 were estimated by qRT-PCR analysis and western blot analysis, respectively. In vivo antitumor activities were investigated by subcutaneous xenograft assay.

Results: The present study firstly demonstrated that the sensitivity of DDP in DDP-resistant A549 (A549/DDP) cells was enhanced when treatment with CB. Moreover, CB combined with DDP significantly weakened the proliferation and increased apoptosis of A549/DDP cells. In addition, the expression level of Bcl-2 was increased, whereas Bax and Caspase- 3 were activated when A549/DDP cells were treated with both drugs. Moreover, after treatment with IGF1 (activator of PI3K/AKT) or PMA (activator of MAPK/ERK) and mixed drugs (CB+DDP), the expressions of P-AKT, P-PI3K, P-MEK1/2 and P-ERK1/2 were increased. Finally, the results of in vivo experiments showed that the combination of DDP and CB significantly reduced the growth of tumors derived from A549/DDP cells.

Conclusions: In summary, the results of this study indicate that the combination of CB and DDP can be considered an effective strategy to increase the sensitivity of DDP-resistant lung cancer cells to DDP by inhibiting the PI3K/AKT and MAPK/ERK pathways.

\section{Introduction}

Up to now, lung cancer is still one of the malignant tumors that endanger human health, and its incidence is increasing year by year worldwide (1-3). Due to the lack of effective early diagnosis methods and unobvious symptoms, most patients are already at an advanced stage when they are discovered and can not be surgically removed. Chemotherapy has become the most commonly used method for clinical treatment of lung cancer (3). Cis-dichlorodiamino platinum [cisplatin (DDP)], a representative reference standard for first-line chemotherapy for lung cancer (4). However, patients with long-term use of cisplatin will develop resistance, which can reduce the survival time and the quality of life of patients (5).

Therefore, it is critical to provide effective methods to improve the sensitivity of DDP resistance for the successful treatment of lung cancer.

Traditional Chinese medicine (TCM) has attracted great attention in the field of drug development due to its low toxicity and high efficiency, especially its application in the treatment of cancer $(6,7)$. Chan-su is a 
traditional Chinese medicine recognized by some Asian countries. It is taken from the dry secretions and parotid glands of Asian toads Bufo gargarizans (8). Cinobufagin (CB), the main active ingredient of Chansu, is approved by the State Food and Drug Administration for the treatment of liver cancer and prostate cancer (9). A deal of evidence has shown that cinobufagin can inhibit the growth and development of cancer (10-12). For example, cell cycle was arrested in $S$ phase and the apoptosis rate was improved when treated with CB in nasopharyngeal carcinoma cells (10). Moreover, Kim et al. found that CB suppressed melanoma cell growth by inhibiting Wnt/ $\beta$-catenin signaling via LEF1 inhibition (11). It was found that IL-6-OPN-STAT3 signaling pathway involved in inhibiting growth and tumorigenesis capability by $\mathrm{CB}$ in osteosarcoma cells (11). It has also been reported that $\mathrm{CB}$ inhibited non-small cell lung cancer (NSCLC) cells growth and promoted cancer apoptosis by inducing the AKT signaling pathway (12). However, the mechanism of CB-mediated resistance to lung cancer is poorly understood, so far only a research has found that $\mathrm{CB}$ can non-competitively reverse chemoresistance of cancer cells through inhibiting the efflux function of P-gp (13). In this paper, we firstly found that the CB can inhibit DDPresistance of $A 549$ lung cancer cells in vitro and in vivo through inhibiting the PI3K/AKT and MEK/ERK pathways

\section{Materials And Methods \\ 2.1 Cell Culture}

The human cancer cell lines A549 and A549/DDP were purchased from the cell bank of the Shanghai Chinese Academy of Sciences Type Culture Collection Committee, and the cells were placed in a constant temperature carbon dioxide cell incubator at $37^{\circ} \mathrm{C}$ and $5 \% \mathrm{CO}_{2}$.

\subsection{CCK-8 assay}

The A549/DDP cells were seeded in a 96-well plate and exposed to CB, DDP, IGF1 and PMA. Cells were subjected to DDP in group DDP, to $C B$ in group $C B$, to $C B$ combined with DDP in group CB+DDP, to IGF1 combined with mix drugs $(C B+D D P)$ in group IGF1+CB+DDP and to PMA combined with mix drugs $(C B+D D P)$ in group $P M A+C B+D D P$.

The A549/DDP cells were subjected to different concentrations of $\mathrm{CB}(0,0.6,1.2,2.5,5,10$ and $20 \mathrm{uM})$ or $\operatorname{DDP}(0,5,10,20,40,80,160$ and $320 \mathrm{uM})$ for $24 \mathrm{~h}$. In the combined group, the concentration of CB was $0.1 \mathrm{uM}$ and that of DDP was $30 \mathrm{uM}$, the level of IGF1 was $100 \mathrm{ng} / \mathrm{mL}$ and PMA was $40 \mathrm{uM}$ and that of mix drugs ( $0.1 \mathrm{uM} \mathrm{CB}+30 \mathrm{uM}$ DDP). Then add the CCK-8 solution to each well and incubate the plate for another $4 \mathrm{~h}$, and finally used the microplate reader to detecte the absorbance at $450 \mathrm{~nm}$ and calculated the cell inhibition rate.

\subsection{Flow cytometry analysis}

The A549/DDP cells were cultured into 6-well plates and placed in a cell incubator for overnight culture, and the cells were divided into three or four groups and treated as above. Then digest cells with 
pancreatin (without EDTA) cells. Cells were stained with AnnexinV-APC for 10 minutes and 7-AAD for 5 minutes in the dark, finally flow cytometry was used for collection and detection.

\subsection{Invasion assay}

The A549/DDP cells were cultured into 6-well plates and placed in a cell incubator for overnight culture, and were allowed to attach overnight in complete growth media. Cells were divided into three groups and treated as above. Before seeding the cells, the coated Matrigel was spread in the upper chamber. The lower chamber was filled with medium containing $10 \% \mathrm{FBS}$. Cells were incubated for $24 \mathrm{~h}$ at $37^{\circ} \mathrm{C}$ and invade through the matrigel. The cells adhering to the lower chamber were fixed with $4 \%$ paraformaldehyde for 30 minutes, stained with $0.5 \%$ crystal violet, and finally photographed with an inverted microscope.

\section{5 qRT-PCR analysis}

According to the manufacturer's instructions, TRIzol reagent was used to extract the total RNA in the cell. The total RNA was then reversely transcribed into cDNA according to the PrimeScript RT Reagent Kit instructions, and finally conducted the quantitative real-time polymerase chain reaction (qRT-PCR) analysis. The PCR primer sequences are as shown in Table 1.

The $\beta$-actin was used as internal reference and the $2^{-\triangle \Delta C t}$ method was used to analyze the relative expression levels of target genes.

\subsection{Western blot analysis}

The A549/DDP cells were cultured into 6-well plates and placed in a cell incubator for overnight culture, and were allowed to attach overnight in complete growth media. Cells were divided into three groups and treated as above. Total protein was extracted and was denatered by boiling water for 10 minutes, then protein concentration was measured by BCA method. Take protein samples for gel electrophoresis, add primary antibodies and incubated overnight at $4^{\circ} \mathrm{C}$. After washing with PBS, membranes incubate the secondary antibody at room temperature, and detect the target protein in the gel imaging system after elution.

\subsection{Subcutaneous xenograft assay}

A total of 15 male BALB/c nude mice (6-8 weeks, 20-22 g) were housed under specific pathogen free (SPF) conditions. After digestion of A549/DDP cells with trypsin, a cell suspension was prepared with PBS and cultured into the groin of nude mice. Randomly grouped according to body weight, the experimental groups were divided into: DMSO group, monotherapy groups (DDP) and a combination treatment group. After the tumor volume grows to $100-200 \mathrm{~mm}^{3}$, DDP and CB were injected intraperitoneally at a dose of $5 \mathrm{mg} / \mathrm{kg}$ and $3 \mathrm{mg} / \mathrm{kg}$ every three days for 51 days, respectively, the tumor size was measured with a vernier caliper, 51 days after the administration, the nude mice were sacrificed, and the tumor tissues were weighed and photographed.

\subsection{Immunohistochemistry}


The mouse tissue sections were deparaffinized with xylene (twice) for 15 minutes and hydrated with an alcohol series ( $100 \%$ twice, $90 \%$ twice, $80 \%$ once). After blocking the sections with $5 \% \mathrm{BSA}$, the sections were incubated with the primary antibody overnight at $4^{\circ} \mathrm{C}$. The next day, the sections were incubated with the secondary antibody at $37^{\circ} \mathrm{C}$, and then treated with $\mathrm{DAB}$ chromogen and hematoxylin at room temperature, and finally observed with an inverted microscope.

\subsection{TUNEL assay}

TUNEL staining was performed to determine apoptosis in each group following the manufacturer's instructions. The sections were deparaffinized with xylene and rehydrated using gradient ethanol. The permeable solution was then added for incubation at $37^{\circ} \mathrm{C}$ for 8 min. After rinsing with PBS, mixed TdT+dUTP were added to the treatment group, dUTP was added to the negative control group, $100 \mu \mathrm{l}$ DNase I was added to the positive control group and then incubated at $25^{\circ} \mathrm{C}$ for $10 \mathrm{~min}$. The slides were then rinsed with PBS. The converter-POD was added and incubated at $37^{\circ} \mathrm{C}$ for $30 \mathrm{~min}$. After washing with PBS, the IDAB substrate was applied on the slides and reacted at $25^{\circ} \mathrm{C}$ for $10 \mathrm{~min}$. The slides were then rinsed with PBS. Hematoxylin was added as a counter stain and sealed with neutral gum. The slides were observed using an optical microscope and the representative images were captured with a digital camera.

\subsection{Statistics analysis.}

All data were expressed as mean \pm SEM. GraphPad Prism 8.0 software was used for statistical analysis. Student's $T$ test was used to compare the two groups, and $P<0.05$ was considered statistically significant.

\section{Results}

\subsection{CB enhanced the inhibitory effect of DDP against A549/DDP cells}

Firstly, the cell viability of A549 and A549/DDP was examined by CCK8 assay in the study. It was found that DDP induced a significant loss of cell viability on the $A 549$ cancer cells, whose $I_{50}$ reached $4.03 \pm 0.29 \mu \mathrm{M}$ (Figure 1A). Then, As shown in Figure 1B and 1C, both CB and DDP exhibited concentration-dependent inhibitory effects on A549/DDP lung cancer cells, whose $\mathrm{IC}_{50}$ reached approximately $1.33 \pm 0.51 \mu \mathrm{M}$ and $30.78 \pm 0.38 \mu \mathrm{M}$, respectively. Moreover, compared to DDP group, the antigrowth effect of CB on the DDP/A549 cancer cells was much significantly stronger, suggesting DDP/A549 cells were more sensitive to $C B$ treatment. Furthermore, the combination inhibitory effect of $\mathrm{CB}$ and DDP was further study. As shown in Figure 1D, the value of $\mathrm{IC}_{50}$ of in combination group exhibited an obvious lower compared with the cells treated with DDP, which revealed that the combination of $C B$ and DDP could significantly inhibit the viability of DDP/A549 cancer cells.

\subsection{CB combined with DDP regulated apoptosis and invasion of DDP/A549 cancer cells}


Secondly, the synergistic effect of CB and DDP on the growth of DDP/A549 cancer cells related to cell apoptosis was further studied. As shown in Figure 2A and 2B, compared to DMSO group, the apoptosis rates were increased in DDP-alone group and combination group. Furthermore, compared to the DDPalone group, the combination effect of $\mathrm{CB}$ and DDP showed the highest apoptosis rate. It is well known that Caspase-3, Bcl-2 and Bax have crucial roles in the execution of apoptosis. Therefore, we further study the expression levels of apoptosis-related proteins by qRT-PCR and western blot. As shown in Figure 2C, compared to the DMSO group, the cells treated with DDP displayed an obvious increased mRNA expression of Caspase-3 and Bax, while decreased mRNA expression of Bcl-2. More importantly, these mRNA expressions of apoptosis-related proteins were further regulated in combination group. (Figure 2C). Subsequently, the results revealed that the expression level of apoptosis-related proteins was a similar result in A549/DDP cancer cells (Figure 2D-E). Metastasis is the leading cause of death in lung cancer patients because of the aggressive ability of cancer cells (14). After $24 \mathrm{~h}$ incubation, cells treated with in DDP or CB united with DDP significantly decreased invasiveness, compared with the DMSO group (Figure $2 \mathrm{H})$. These results demonstrated that CB united with DDP induced apoptosis and suppressed DDP/A549 cancer cells invasion. Furthermore, the expression of resistance-related gene in A549/DDP cells was performed. As shown in Figure $2 \mathrm{C}$ and $2 \mathrm{E}-\mathrm{G}$, compared to the control group, the mRNA and protein expression of the multi-drug resistant protein 1 (MRP1) were downregulated in DDP-alone group and CB united with DDP group, respectively.

\subsection{CB enhances the inhibitory effect of DDP on DDP/A549 cancer cells through PI3K/AKT pathway and MAPK/ERK pathway}

Evidence has revealed that drug resistance is directly involved in regulation of intrinsic pathway or extrinsic pathway (15). Recent studies revealed the key roles of PI3K/AKT pathway and MAPK/ERK pathways to chemotherapy resistance (16-19). To explore whether CB affect A549/DDP growth by $\mathrm{PI3K} / \mathrm{AKT}$ and MAPK/ERK pathways. After treatment with IGF1 (activator of PI3K/AKT) or PMA (activator of MAPK/ERK) and mixed drugs (CB+DDP), as shown in Figure 3A-3C, the cell viability and apoptosis rate of A549/DDP cells were increased and decreased compared with those in the single mixed drug treatment group, respectively. After pretreatment with IGF1, A549/DDP cells were incubated with the mixed drugs (CB+DDP). In these cells, the mRNA and protein expressions of P-AKT and P-PI3K were significantly increased compared with those in the single mixed drug treatment group, and the expression of P-MEK1/2 and P-ERK1/2 was also changed. Similarly, A549/DDP cells were incubated with PMA and mixed drugs successively; the expression levels of P-MEK1/2 and P-ERK1/2 were significantly increased compared to those in the mixed drug treatment group alone. More interestingly, A549/DDP cells were incubated with IGF1 or PMA, the mRNA and protein expression of the MRP1 were upregulated, suggesting that PI3K/AKT and MAPK/ERK pathways may be related to MRP1. All data concluded that CB combined with DDP suppressed A549/DDP cells growth through the PI3K/AKT and MAPK/ERK pathways.

\subsection{CB united with DDP inhibits the growth of A549/DDP cells in vivo}


We further investigated the synergistic effect of in CB and DDP drug combination group in vivo. We established the subcutaneous ectopic tumor formation model by injecting A549/DDP cells into nude mice to evaluate the combined anti-tumor effect of CB and DDP. The results showed that compared with the single drug groups, the lung tumor volume in the combination group was significantly lower, which suggested $C B$ united with DDP could be an effect strategy for lung cancer treatment (Figure 4A-4B). Moreover, Immunohistochemical staining indicated that the densities of MRP1, P-MEK1/2 and P-PI3K were significantly more reduced in the combination group compared with that in single drug groups (Figure 4C-4D). Furthermore, the results from the TUNEL assay also revealed the apoptosis rates in the combined drug group were significantly elevated (Figure 4E-4F). All in all, these results indicate that DDP combined with CB had a good anti-tumor effect in vitro.

\section{Discussion And Conclusion}

With the rapid development of therapeutic strategies, the prognosis for patients with advanced lung cancer has been improved, but most patients still are poor mainly due to the rapid progress chemotherapy-resistance $(1,2)$. At present, DDP is considered to be a commonly used chemotherapy drug for lung cancer patients. However, long-term use of DDP will gradually increase the risk of drug resistance and may have side effects, resulting in reduced therapeutic effects and severely affecting the health of patients $(4,5)$. The study found that the 5 -year survival rate of lung cancer patients treated with DDP is only $4-17 \%(20)$. At the same time, studies have found that the combination of DDP and other drugs can significantly reduce drug resistance, indicating that combination drugs may be a potential treatment strategy to reduce single-drug resistance $(21,22)$.

According to previous reports, $\mathrm{CB}$ has good anti-tumor activity against a variety of cancers, including gastric cancer, lung cancer, cachexia, breast cancer, osteosarcoma and pancreatic cancer (23). However, there are rare studies demonstrated that $\mathrm{CB}$ against drug-resistant-cancers. In present study, a combined treatment strategy of CB and DDP to study the synergy of anti-A549/DDP lung cancer cells was established. The results showed that the cell viability was reduced in combination group, compared with the A549/DDP cells treated with DDP, which suggested that CB may enhance the sensitivity of A549/DDP cells to DDP. However, the potential combination mechanism is not yet clear and requires further research. Further study found that the apoptosis rate of the A549/DDP cells in the combination group was significantly elevated, suggesting that CB could enhance the apoptosis activity of DDP in A549/DDP cells. The results of qRT-PCR and western blotting indicated that the ability to increase sensitivity of CB to the treatment of DDP may be mainly related to elevated apoptosis-related proteins expressions. In general, tumor cells have greater adaptability to the environment and fortissimo independent survival, and can infinitely invade. Our results showed that A549/DDP cells treated with in CB united with DDP significantly decreased invasiveness, showing that CB united with DDP reduces invasion of lung cancer cells. It has been reported in the literature that drugs can reverse chemoresistance of cancer cell that might be related to resistance-related genes, including MRP1. qRT-PCR analysis and western blotting assay indicated that CB did down-regulate the expression of MRP1 in in A549/DDP cells. 
Obviously, PI3K/AKT and MAPK/ERK pathways are important components that regulate cancer cell proliferation, migration, and metastasis. In addition, many studies have shown that DDP resistance is related to altered cancer cell signaling pathways, including PI3K/AKT and MAPK/ERK pathways $(24,25)$. Therefore, we investigated whether the effect of $C B$ in promoting DDP sensitivity is related to PI3K/AKT and MAPK/ERK signaling pathways. In our study, qRT-PCR and western blot assays were performed to investigated the activity of $\mathrm{CB}$ on the levels of the PI3K/AKT and MAPK/ERK pathway-related proteins. Our data found that CB inhibited AKT, PI3K, MEK1/2 and ERK1/2 in the mRNA and protein levels, respectively. However, when A549/DDP cells were incubated with PMA or IGF1 and mixed drugs, these expressions were increased. Moreover, the results showed that inhibition of apoptosis rate was inhibited by adding PMA or IGF1, suggesting that CB is through PI3K/AKT and MAPK/ERK signaling pathways sensitized DDP to DDP-resistant A549/DDP cells.

Based on the above in vitro experimental results, we further studied the anti-lung cancer effect of $C B$ combined with DDP on A549/DDP cells in vivo. The results showed that CB increased the inhibitory effect of DDP on tumor growth, and the tumor volume was the smallest in combination group. In addition, the levels of MRP1, P-MEK1/2 and P-PI3K were also the lowest in the combined group. In addition, we proved that the apoptosis rate was significantly increased after CB and DDP combined treatment. Taken together, it is concluded that CB significantly enhances the anti-tumor effect of DDP.

In conclusion, we found that $\mathrm{CB}$ can increase the sensitivity of chemotherapy-resistant lung cancer cells to DDP by inhibiting the PI3K/AKT and MAPK/ERK pathways, which may provide a theoretical basis for the treatment of DDP-resistant lung cancer.

\section{Declarations}

Funding: This work was supported by the national natural science fund committee [grant numbers 81702975$]$.

Acknowledgements: Not applicable.

Author Contributions: Guangxin Zhangand Xueshibojie Liuperformed the experiment, analyzed data, and drafted the paper. Yicun Wangand Chengyan Jinperformed the experiments. Zhengyang Lu and Hao Yang analyzed data, and drafted the paper. Ti Tongorganized and designed the study. All authors read and approved of the final manuscript.

\section{Compliance with Ethical Standards}

Conflict of interest The authors declare that they have no confict of interest.

Ethics approval and consent to participate: The study was approved by the Ethics Committee of General Hospital of Second Hospital of Jilin University.

Consent to participate: All authors have confrmed their participation in this study. 
Consent to publish: All authors have agreed to publish this manuscript.

\section{References}

1. Pierret T, Toffart AC, Giaj Levra M, Moro-Sibilot D, Gobbini E (2020) Advances and therapeutic perspectives in extended-stage small-cell lung cancer. Cancers (Basel) 12:3224-3242

2. Ocariz-Diez M, Cruellas M, Gascon M, Lastra R, Martinez-Lostao L, Ramirez-Labrada A, Pano JR, Sesma A, Torres I, Yubero A, Pardo J, Isla D, Galvez EM (2020) Microbiota and lung cancer. Opportunities and challenges for improving immunotherapy efficacy. Front Oncol 10:568939568947

3. Yan HQ, Huang XB, Ke SZ, Jiang YN, Zhang YH, Wang YN, Li J, Gao FG (2014) Interleukin 6 augments lung cancer chemotherapeutic resistance via ataxia-telangiectasia mutated/NF-kappaB pathway activation. Cancer Sci 105:1220-1227

4. Sun Y, Wu YL, Li LY, Liao ML, Jiang GL, Kim ES, Douillard JY, Milenkova T (2011) [Efficacy and safety of gefitinib or docetaxel in Chinese patients with locally advanced or metastatic non-small cell lung cancer (NSCLC) who had failed previous platinum-based first-line chemotherapy]. Zhonghua Zhong Liu Za Zhi 33:377-380

5. Ke B, Wei T, Huang Y, Gong Y, Wu G, Liu J, Chen X, Shi L (2019) Interleukin-7 resensitizes non-sSmallcell lung cancer to cisplatin via inhibition of ABCG2. Mediators Inflamm 2019:7241418-7241436

6. Xu H, Zhao X, Liu X, Xu P, Zhang K, Lin X (2015) Antitumor effects of traditional Chinese medicine targeting the cellular apoptotic pathway. Drug Des Devel Ther 9:2735-2744

7. Xia J, Chen J, Zhang Z, Song P, Tang W, Kokudo N (2014) A map describing the association between effective components of traditional Chinese medicine and signaling pathways in cancer cells in vitro and in vivo. Drug Discov Ther 8:139-153

8. Wang D, Ru W, Xu Y, Zhang J, He X, Fan G, Mao B, Zhou X, Qin Y (2014) Chemical constituents and bioactivities of Colla corii asini. Drug Discov Ther 8:201-207

9. Zhou Y, Hou J, Long H, Zhang Z, Lei M, Wu W (2020) Design, synthesis and anti-tumor activities of carbamate derivatives of cinobufagin. Steroids 164:108749-108760

10. Pan Z, Luo Y, Xia Y, Zhang X, Qin Y, Liu W, Li M, Liu X, Zheng Q, Li D (2020) Cinobufagin induces cell cycle arrest at the $S$ phase and promotes apoptosis in nasopharyngeal carcinoma cells. Biomed Pharmacother 122:109763

11. Kim GH, Fang XQ, Lim WJ, Park J, Kang TB, Kim JH, Lim JH (2020) Cinobufagin suppresses melanoma cell growth by inhibiting LEF1. Int J Mol Sci 21:6706-6719

12. Zhang C, Ma K, Li WY (2019) Cinobufagin suppresses the characteristics Of osteosarcoma cancer cells by inhibiting the IL-6-OPN-STAT3 pathway. Drug Des Devel Ther 13:4075-4090

13. Yuan Z, Shi X, Qiu Y, Jia T, Yuan X, Zou Y, Liu C, Yu H, Yuan Y, He X, Xu K, Yin P (2017) Reversal of Pgp-mediated multidrug resistance in colon cancer by cinobufagin. Oncol Rep 37:1815-1825 
14. Zhao YY, Jia J, Zhang JJ, Xun YP, Xie SJ, Liang JF, Guo HG, Zhu JZ, Ma SL, Zhang SR (2020) Inhibition of histamine receptor $\mathrm{H} 3$ suppresses the growth and metastasis of human non-small cell lung cancer cells via inhibiting PI3K/Akt/mTOR and MEK/ERK signaling pathways and blocking EMT.Acta Pharmacol Sin1-10

15. BeLow M, Osipo C (2020) Notch signaling in breast Cancer: A role in drug resistance. Cells 9:22042223

16. Ozates NP, Sogutlu F, Lerminoglu F, Demir B, Gunduz C, Shademan B, Avci CB (2020) Effects of rapamycin and AZD3463 combination on apoptosis, autophagy, and cell cycle for resistance control in breast cancer.Life Sci:118643-118652

17. Li C, Feng S, Chen L (2020) MSC-AS1 knockdown inhibits cell growth and temozolomide resistance by regulating miR-373-3p/CPEB4 axis in glioma through PI3K/Akt pathway. Mol Cell Biochem. $10.1007 / \mathrm{s} 11010-020-03937-x$

18. Zheng S, Shu Y, Lu Y, Sun Y (2020) Chloroquine combined with imatinib overcomes imatinib resistance in gastrointestinal stromal tumors by inhibiting autophagy via the MAPK/ERK Pathway. Onco Targets Ther 13:6433-6441

19. Pratt EC, Isaac E, Stater E, Yang G, Ouerfelli O, Pillarsetty NVK, Grimm J (2020) Synthesis of novel PET tracer (124)I-trametinib for MAPK/ERK kinase distribution and resistance monitoring. J Nucl Med 241901. doi:10.2967/jnumed.120

20. Hirsch FR, Scagliotti GV, Mulshine JL, Kwon R, Curran WJ Jr, Wu YL, Paz-Ares L (2017) Lung cancer: current therapies and new targeted treatments. Lancet 389:299-311

21. Huang P, Du SX (2020) Puerarin enhances the anti-tumor effect of cisplatin on drug-resistant A549 cancer in vivo and in vitro through activation of the Wnt Signaling Pathway. Cancer Manag Res 12:6279-6289

22. Chen Y, Hong C, Chen X, Qin Z (2020) Demethoxycurcumin increases the sensitivity of cisplatinresistant non-small lung cancer cells to cisplatin and induces apoptosis by activating the caspase signaling pathway. Oncol Lett 20:209-217

23. Zhang L, Huang X, Guo T, Wang H, Fan H, Fang L (2020) Study of cinobufagin as a promising anticancer agent in uveal melanoma through intrinsic apoptosis pathway. Front Oncol 10:325-334

24. Shu XR, Wu J, Sun H, Chi LQ, Wang JH (2015) PAK4 confers the malignance of cervical cancers and contributes to the cisplatin-resistance in cervical cancer cells via PI3K/AKT pathway. Diagn Pathol 10:177-186

25. Paucarmayta A, Taitz H, McGlorthan L, Casablanca Y, Maxwell GL, Darcy KM, Syed V (2020) Progesterone-calcitriol combination enhanced cytotoxicity of cisplatin in ovarian and endometrial cancer cells in vitro. Biomedicines 8:73-99

\section{Tables}

Due to technical limitations, table 1 is only available as a download in the Supplemental Files section. 


\section{Figures}
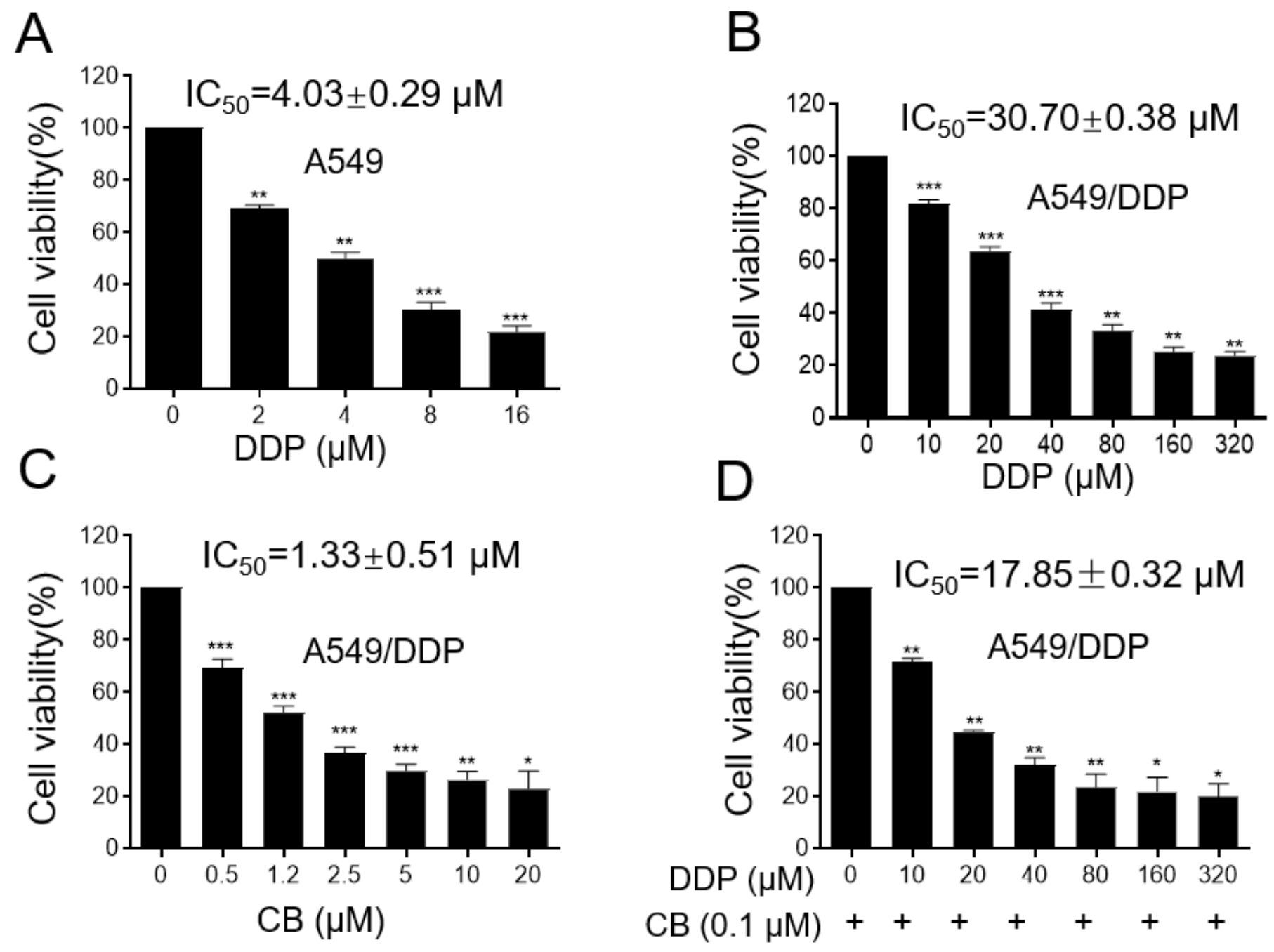

Figure 1

CB united with DDP can reduce the cell viability of DDP/A549 lung cancer cells. (A) The cell viability of A549 cells were detected with different concentrations of DDP for $24 \mathrm{~h}$. (B) The cell viability of A549/DDP cells were detected with different concentrations of CB (C) and DDP for $24 \mathrm{~h}(\mathrm{D})$. The cell viability of A549/DDP cells were treated with $0.1 \mu \mathrm{M} \mathrm{CB}$ combined with different concentrations of DDP for $24 \mathrm{~h}$. Compare DMSO group, $*, p<0.05, * \star, p<0.01, * \star *, p<0.001$ 
A
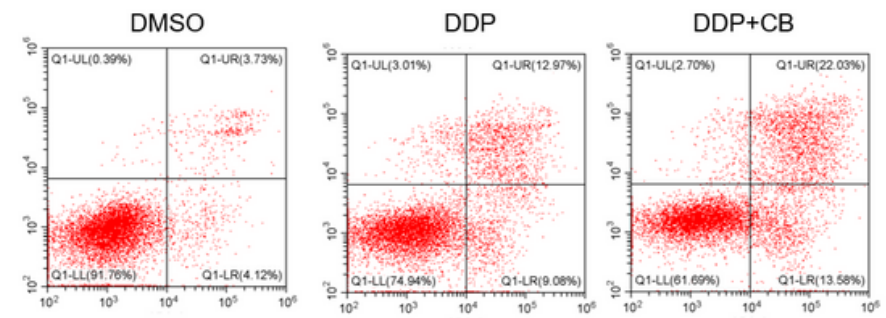

B
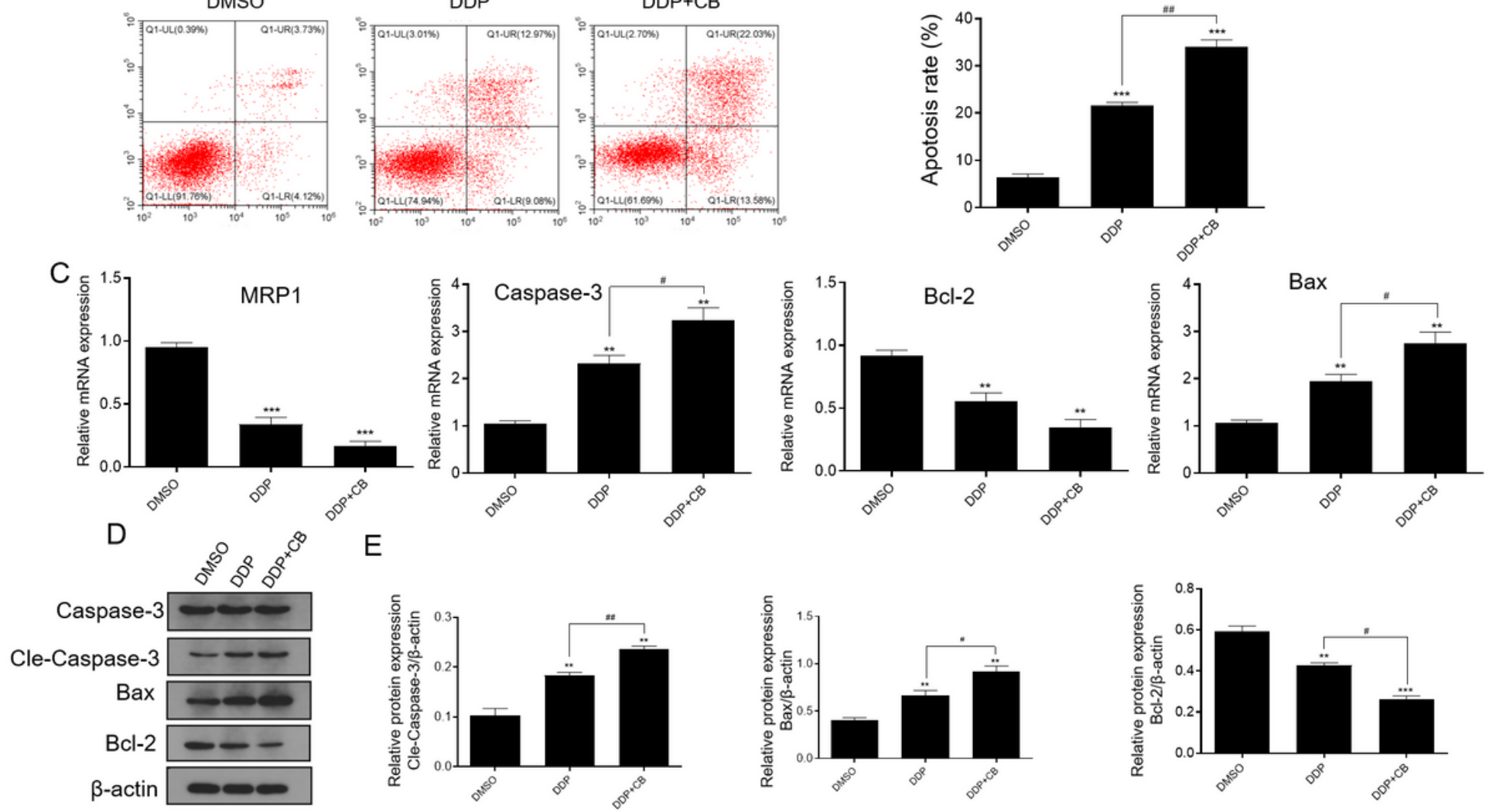

E
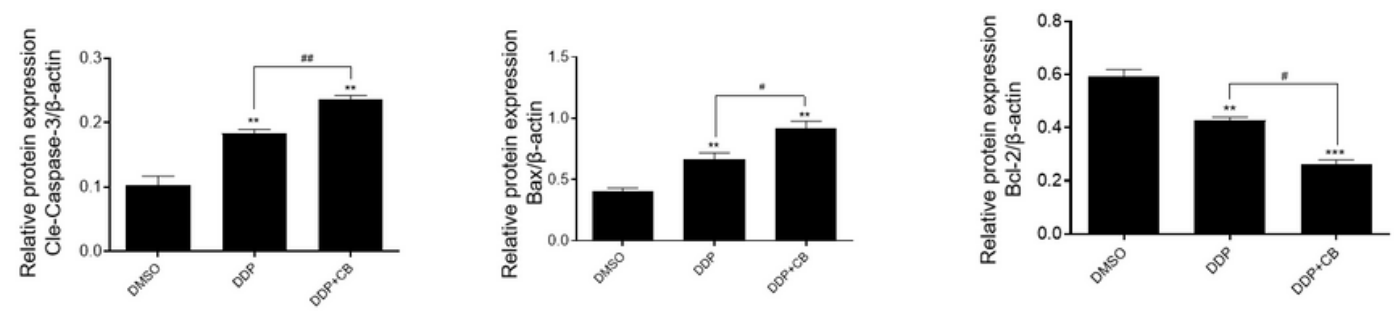

$\mathrm{F}$

G

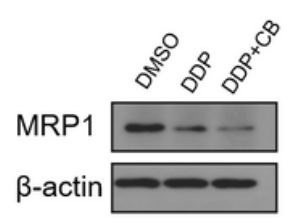

DDP

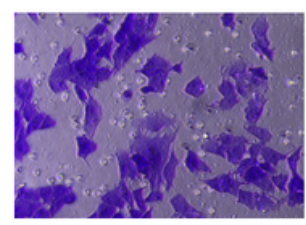

$\mathrm{DDP}+\mathrm{CB}$

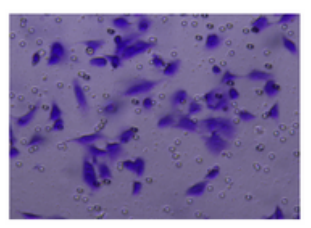

DMSO

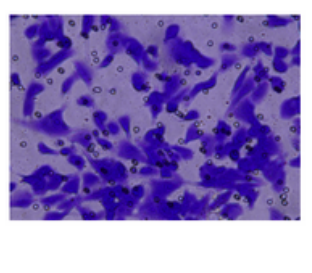

\section{Figure 2}

CB united with DDP increased apoptosis and reduced invasion. (A-B) The apoptosis rate of A549/DDP cells were treated with $0.1 \mu \mathrm{M}$ CB combined with $30 \mu \mathrm{M}$ DDP for $24 \mathrm{~h}$. (C) The mRNA expression of MRP1, Caspase-3, Bcl-2 and Bax on A549/DDP cells were treated with 0.1 $\mu \mathrm{M}$ CB combined with $30 \mu \mathrm{M}$ DDP for 24 h. (D-G) The protein expression of Caspase-3, Bcl-2, Bax and MRP1 on A549/DDP cells were treated with $0.1 \mu \mathrm{M}$ CB combined with $30 \mu \mathrm{M}$ DDP for $48 \mathrm{~h}$. $(\mathrm{H})$ The invasion activity of A549/DDP cells were treated with $0.1 \mu \mathrm{M}$ CB combined with $30 \mu \mathrm{M}$ DDP for $24 \mathrm{~h}$. Compare DMSO group, **, $\mathrm{p}<0.01, * \star \star$, $\mathrm{p}<0.001$; Compare DDP group, \#, $\mathrm{p}<0.05$, \#\#, $\mathrm{p}<0.01$. 
A

B
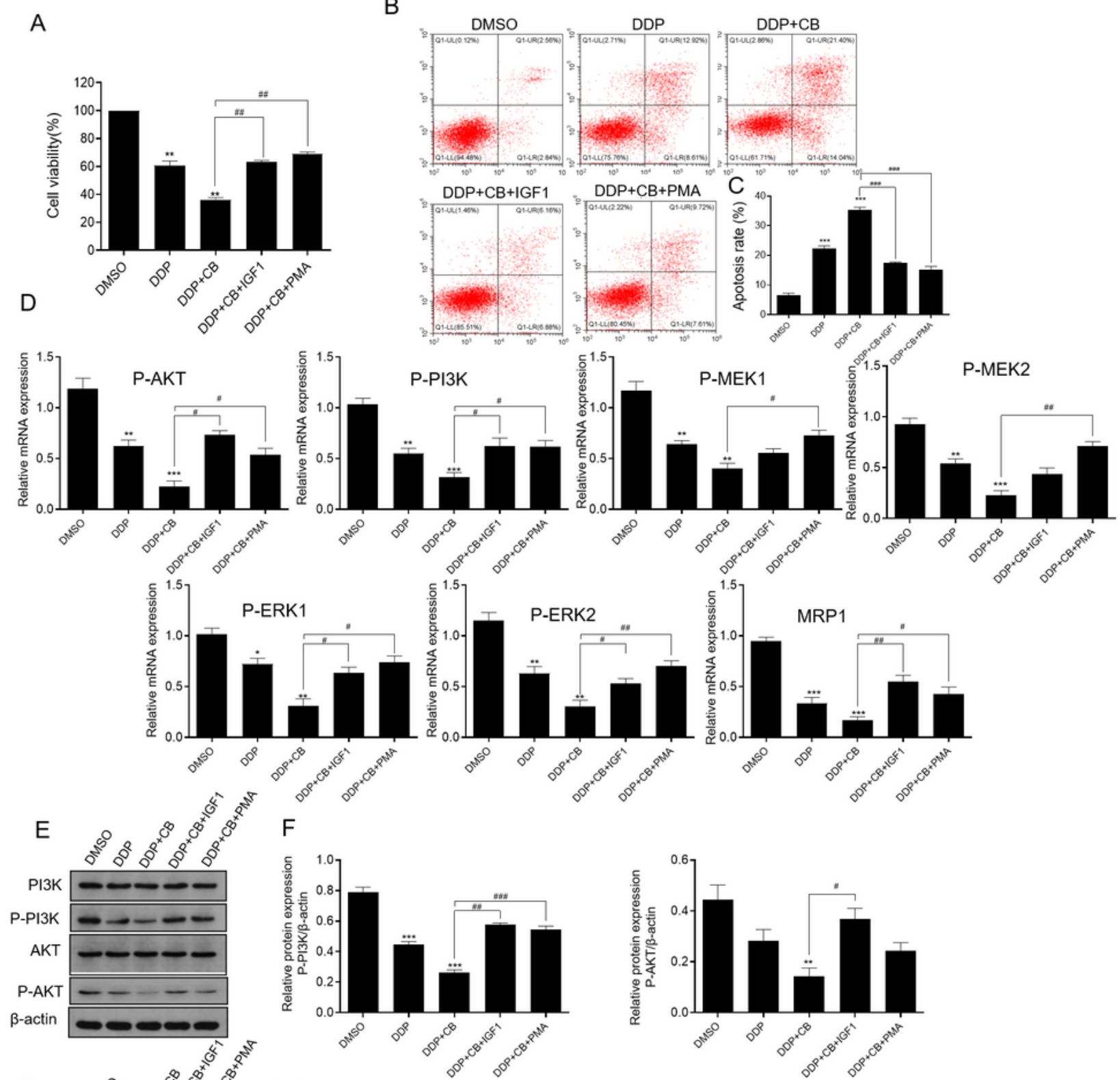

$\mathrm{F}$
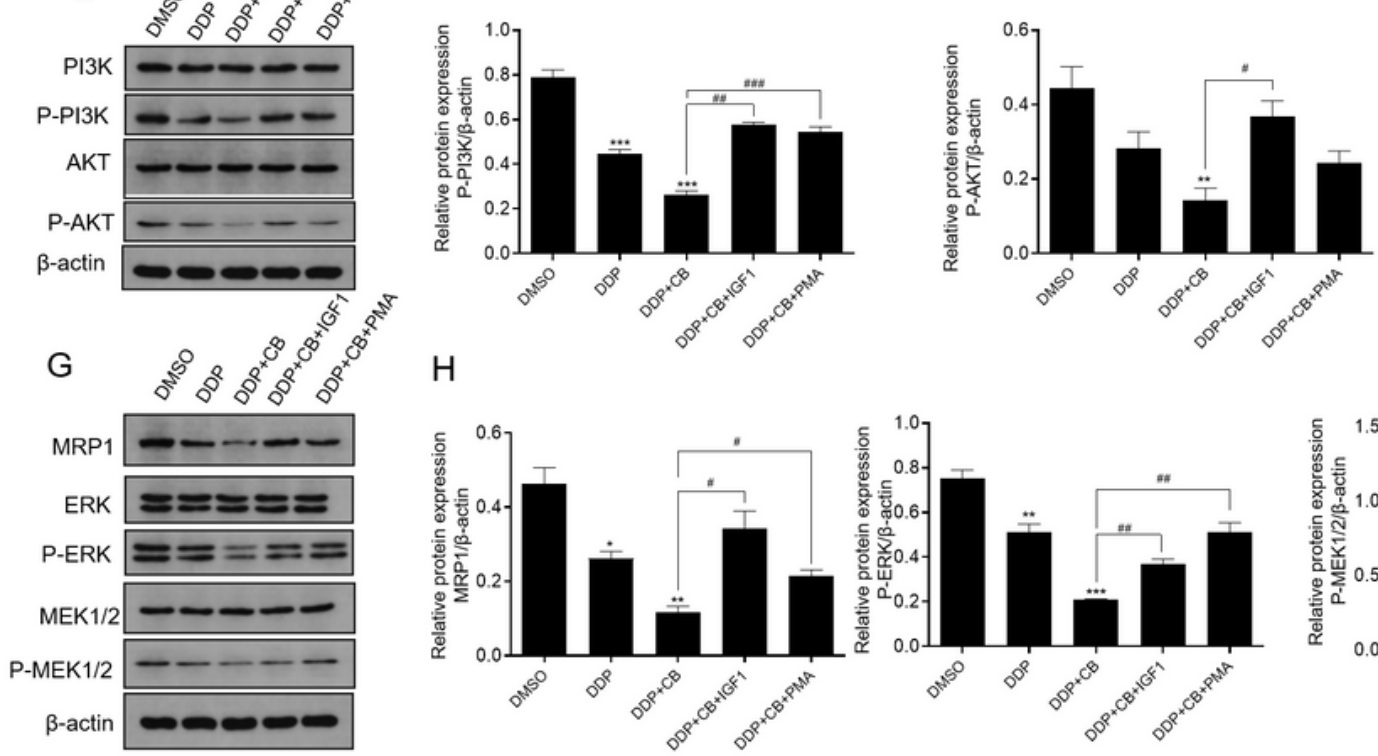

$\mathrm{H}$
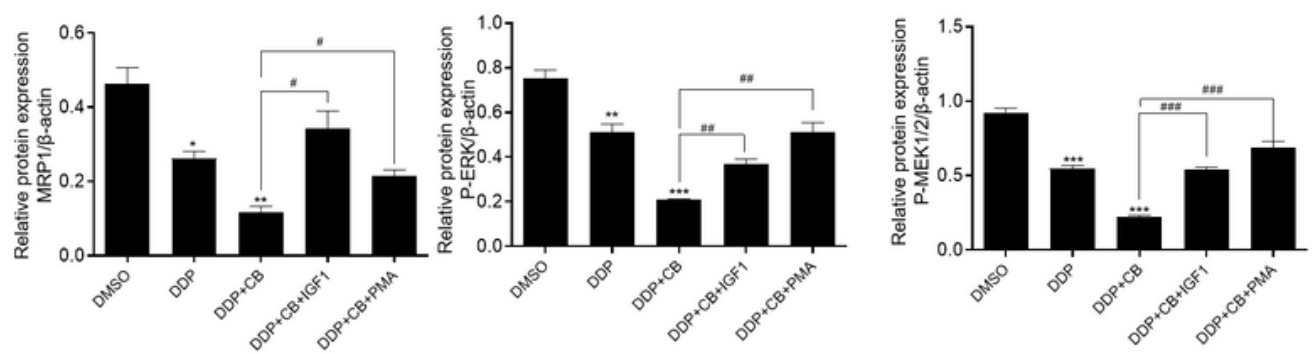

\section{Figure 3}

CB enhances the inhibitory effect of DDP on DDP/A549 cancer cells through PI3K/AKT pathway and MAPK/ERK pathway. (A) The cell viability of A549/DDP cells were detected with IGF1or PMA and mixed drugs (CB+DDP). (B) The apoptosis rate of A549/DDP cells were treated with IGF1or PMA and mixed drugs (CB+DDP). (C-H) The mRNA and protein expression of P-AKT, P-PI3K, P-MEK1/2, P-ERK1/2 and 
MRP1 on A549/DDP cells were treated with IGF1or PMA and mixed drugs (CB+DDP). Compare DMSO group, ${ }^{*}, p<0.05,{ }^{*}, p<0.01$, ${ }^{* *}, p<0.001$; Compare DDP group, $\#, p<0.05, \# \#, p<0.01, \# \# \#, p<0.001$.

A

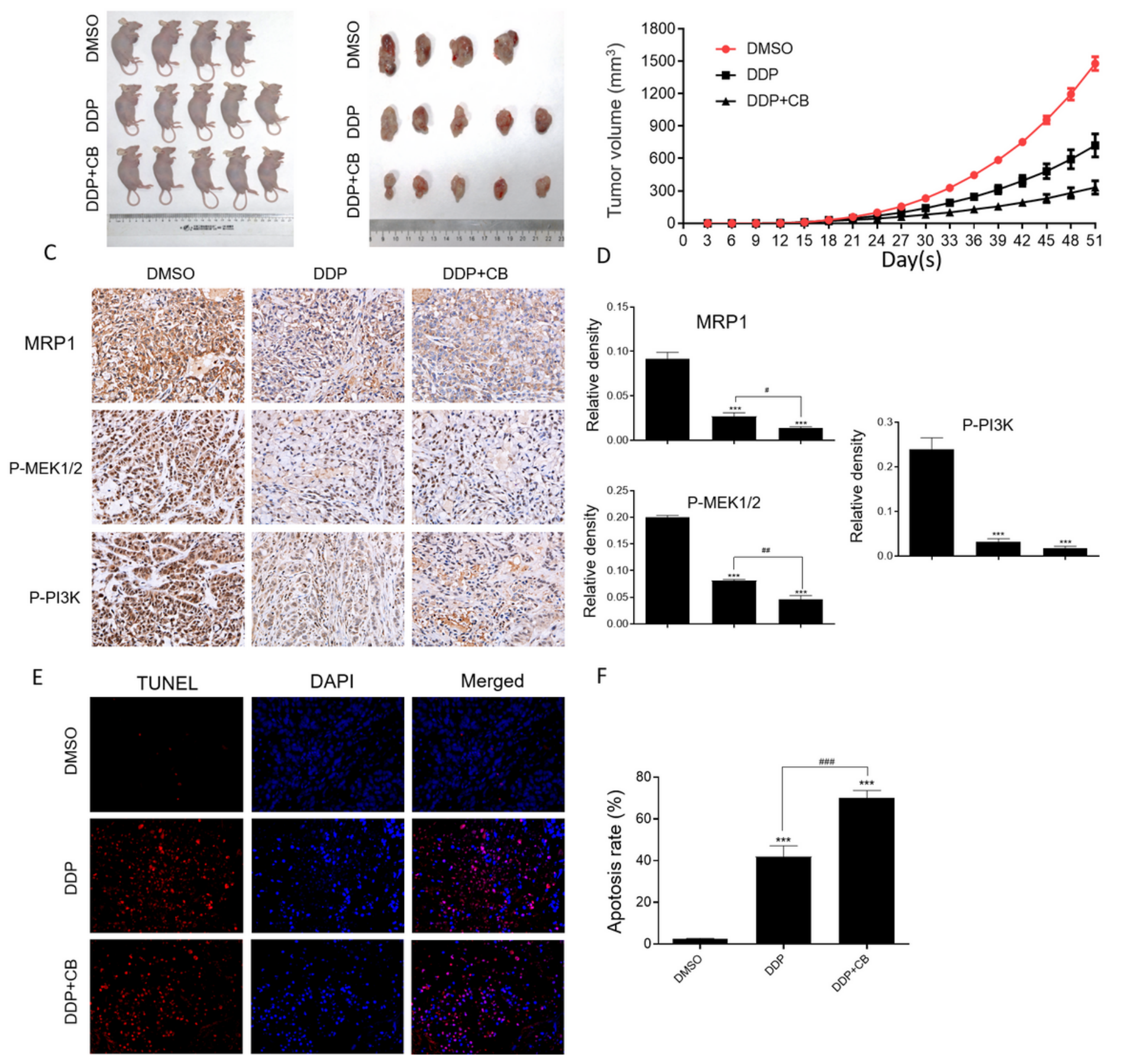

\section{Figure 4}

CB united with DDP inhibits the proliferation of A549/DDP cells in vivo. (A) The qualitative images and quantitative analysis (B) of tumor volumes after the of A549/DDP tumor-bearing mice treated with by $C B$, DDP, and combination of CB and DDP. (C-D) The qualitative (C) and quantitative (D) determination of MRP1, P-MEK1/2 and P-PI3K expression in tumor tissues by immunohistochemical staining. (E-F) The 
qualitative $(E)$ and quantitative $(F)$ determination of apoptosis in tumor tissues. Compare DMSO group, $\star \star \star, p<0.001$; Compare DDP group, \#, p<0.05, \#\#\#, p<0.001.

\section{Supplementary Files}

This is a list of supplementary files associated with this preprint. Click to download.

- Table1.tif 